\title{
Using the Watershed Transform for Iris Detection
}

\author{
Maria Frucci ${ }^{1}$, Michele Nappi ${ }^{2}$, Daniel Riccio ${ }^{3}$, and Gabriella Sanniti di Baja ${ }^{1}$ \\ ${ }^{1}$ Istituto di Cibernetica "E. Caianiello", CNR \\ via Campi Flegrei 34, 80078, Napoli \\ ${ }^{2}$ Università degli Studi di Salerno, \\ via Ponte Don Melillo, 84084 Fisciano (Salerno) Italy \\ ${ }^{3}$ Università degli Studi di Napoli Federico II, \\ via Cintia 21, 80126, Napoli \\ m.frucci@cib.na.cnr.it, mnappi@unisa.it, daniel.riccio@unina.it, \\ g.sannitidibaja@cib.na.cnr.it
}

\begin{abstract}
Iris biometric systems are of interest for security applications. In this respect, iris segmentation has a key role, as it must be fast and accurate. In this paper, we present a new watershed based approach for iris segmentation in color images. The watershed transform is used in two distinct phases of iris segmentation: it is first used to obtain a preliminary segmentation, which constitutes the input to a circle fitting procedure; then, it is used together with the portion of the input image resulting after circle fitting to identify more precisely the pixels actually belonging to the iris. The experimental results show that the suggested approach is effective with respect to both location accuracy and computational complexity.
\end{abstract}

Keywords: Biometrics, iris detection, watershed transformation, circle fitting.

\section{Introduction}

We are often requested to prove our identity. This is the case, for example, to enter places/buildings for which a controlled access has been set, or when we are traveling and cross a border. In any case, the correct authentication of individuals is very important for our security. A typical example is to prevent the impersonation, i.e., to prevent that somebody claims to be somebody else. Nowadays, rather than rely on conventional printed documents such as the passport to prove our identity, the general trend is to use biometrics, such as fingerprints, face, iris, ear and voice, since these features uniquely identify an individual.

A number of papers presenting techniques that involve different biometric features are available in the recent literature. In turn, recognition systems based on a single biometric or on the combination of a number of biometrics have been developed. Iris recognition is generally recognized as one of the most powerful biometric techniques and has been used in different applications, such as physical access control, employee identification, and information systems security. The interest in this biometric is witnessed by a successful international initiative called Noisy Iris Challenge Evaluation, which has already been held twice (NICE I [1] and NICE II [2]). 
Iris recognition offers a number of advantages. For example, differently from fingerprints for which contact between finger and device is necessary, the iris can be scanned from a few centimeters up to a few meters away; iris recognition can be accomplished also for blind individuals since recognition is based on the iris pattern, not on the sight of the individual; iris recognition is not invasive due to the use of commercial iris cameras that use infrared light and do not cause any trouble to the subject; finally, provided that there are no injuries, the iris remains stable for the whole life.

Iris recognition systems mainly operate in the near infrared (NIR) spectrum (e.g., $[3,4]$ ), or by using visible wavelength light (e.g., [5]). The whole iris recognition process can be described as consisting of the following steps: image capture, eye localization, iris segmentation, noise detection, normalization, feature extraction, matching. In this paper, we focus on iris segmentation and will describe a method based on watershed transformation that allows us to obtain a correct iris segmentation at a low computational cost. We will test our method on the UBIRIS database [6], which includes heavily degraded iris image and is the database that has been used for the two NICE events.

\section{Preliminaries}

In this paper, we work with RGB color images with size $800 \times 600$ taken from the UBIRIS database [6]. An image $I$ from this database is used as running example in this paper. Preliminarily, to reduce the overall computation time, images are resized by using a scaling down method based on linear interpolation with reduction factor 0.25 . Thus, we actually process in place of $I$ an image $I r$ with size $200 \times 150$ (Fig. 1 left). Then, a median filter with window size $7 \times 7$ is applied to the image $I r$ to remove noise and smooth sharper color differences (Fig. 1 middle) and the Sobel operator is used to build the gradient image (Fig. 1 right). The above processes have been implemented by using standard OpenCV libraries.
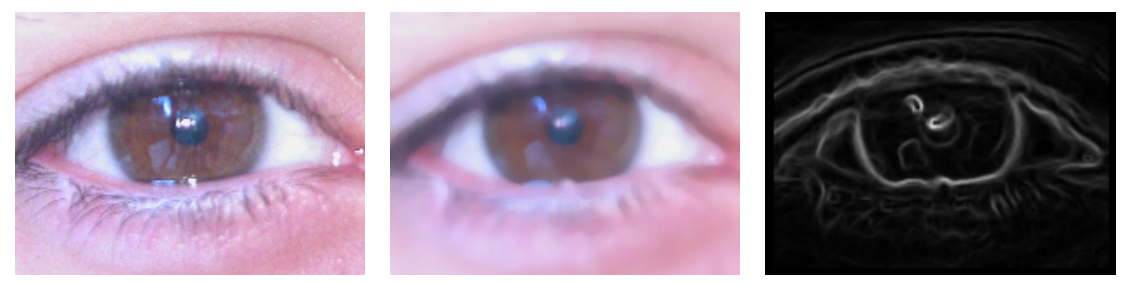

Fig. 1. The $200 \times 150$ resized image $I r$, left, image smoothed by median filter, middle, gradient image, right

For an RGB image, the colors can be interpreted as points in a 3D Cartesian space where the coordinate axes are red, green and blue. The three coordinates of black and white are respectively $(0,0,0)$ and $(255,255,255)$. Since the coordinates of any color point are in the range $[0,255]$, all possible colors are placed within a cube. See Fig. 2. 


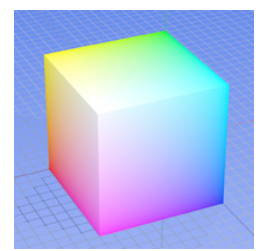

Fig. 2. Colors represented as points in a cube

For iris segmentation we resort to the watershed transformation that has been introduced for gray-level images in [7]. The watershed transformation is a well known technique to partition an image into a number of regions in such a way that each region is homogeneous with respect to a given property, while the union of any two adjacent regions is not. The partition is obtained by applying region growing to a suitable set of seeds. Generally, the seeds are detected as the regional minima in the gradient image of the input image, while region growing takes into account both the distances from the seeds and a specific homogeneity criterion.

The landscape paradigm is often used to explain the mechanism of the watershed transformation. According to this paradigm, the gradient image is interpreted as the top surface of a 3D binary landscape, which can be obtained by considering for each pixel of the gradient image with planar co-ordinates $(x, y)$, the relative gradient value as representing the third co-ordinate $z$, that is the height in position $(x, y)$ of the landscape. In this way, high gradient values correspond to mountains and low gradient values to valleys. Peaks of mountains and pits of valleys are respectively identified by the connected components of pixels with locally higher and locally lower gradient values.
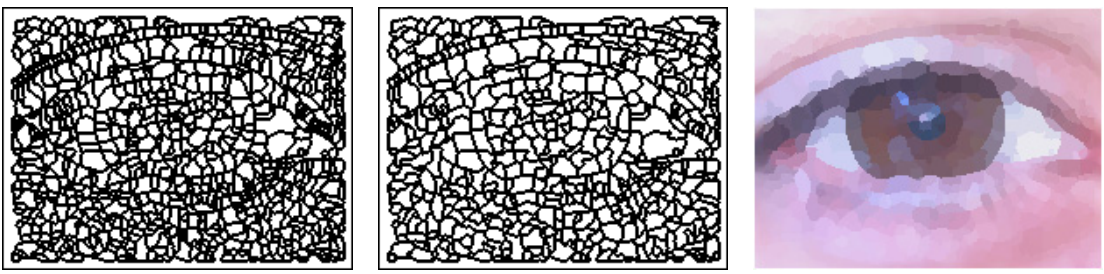

Fig. 3. Watershed lines before merging, left, watershed lines after merging, middle, color quantized image, right

In this paper, the watershed transform $W$ is computed by using the topographical distance watershed transformation algorithm [8]. The image $I r$ is divided into a number of disjoint regions $R_{i}$ whose union completely recovers $I r$. See Fig. 3 left, where for a better visualization only the watershed lines superimposed over a white background are shown.

We build a color quantized version $I q$ of the image $I r$ by assigning to all pixels in the same partition region $R_{i}$ of $I r$ a unique representative color $c_{i}$. The red, green and blue components of $c_{i}$ are computed as the arithmetic means of the red, green and blue components of the colors of all pixels in $R_{i}$. 
Watershed lines generally border in a satisfactory way the regions into which the image is partitioned. However, if all the regional minima detected in the gradient image are taken as seeds for the growing process, a huge number of regions is obtained, which is likely to make hard the successive process aimed at assigning the various regions to the foreground or the background. This problem can be alleviated by reducing the number of seeds or by merging adjacent regions that do not differ too drastically from each other as far as the homogeneity is concerned. Here, we follow the latter strategy and merge adjacent regions whose representative colors are sufficiently similar. In particular, for any region $R_{i}$ the adjacent regions whose representative colors have Euclidean distance from $c_{i}$ smaller than an a priori fixed threshold $\vartheta$, set to 50 in the work presented in this paper, are considered as candidates for merging. Merging of $R_{i}$ is actually accomplished with the adjacent region $R_{j}$ for which the distance between the representative colors $c_{i}$ and $c_{j}$ has the minimum value. After merging, the representative colors of the partition regions are updated. The watershed lines resulting after merging and the updated quantized image are shown in Fig. 3 middle and Fig. 3 right respectively. For the running example, merging allows us to reduce from 879 to 542 the number of regions in the watershed partitioned image. This number could be furthermore reduced by applying again merging or by using a larger tolerance. However, we prefer not to risk missing some parts of the iris boundary, which might happen especially when analyzing light eyes.

\section{Segmentation}

The watershed transform $W$ is used in two phases of the iris detection process. $W$ is first used to obtain a preliminary segmentation, which constitutes the input to a circle fitting procedure. Then, the portion of the input image resulting after circle fitting and the watershed transform $W$ are used together to more precisely identify the pixels actually belonging to the iris.

\subsection{Preliminary Segmentation}

Our purpose is to build a suitable binary version of the watershed transform $W_{(0,1)}$ by ascribing each of its regions to either the foreground or the background. The binary version of $W$, even if it does not provide the desired iris segmentation, makes it possible to detect sufficiently large portions of the iris boundary. Then, fitting a circle in the binary version of $W$ is expected to correctly identify the iris boundary.

For each partition region $R_{i}$, let $d b_{i}$ and $d w_{i}$ be the Euclidean distances in the RGB space of the representative color $c_{i}$ from black and white colors, respectively. Moreover, let $d b$ and $d w$ be the arithmetic means of the distances from black and white colors of all representative colors. Finally, let $d b w$ denote the distance between black and white colors. Such a distance is $d b w=441.6730$.

In principle, regions whose representative colors have a distance from black (white) smaller than $d b(d w)$ could be assigned to the foreground (background) of $W_{(0,1)}$ in consideration that the region occupied by iris and pupil (sclera and eyelids) 
is generally characterized by a color closer to black (white) than to white (black). However, depending on the values of the two arithmetic means $d b$ and $d w$ there might be regions of $W$ that remain unassigned in $W_{(0,1)}$ since their representative colors have distances from both black and white that are larger than the two respective arithmetic means. This is the case when it results $d b+d w<d b w$. In turn, there might be regions for which assignment to the foreground or the background is ambiguous due to the fact that their representative colors have distances smaller than or equal to the respective arithmetic means from both black and white. This is the case when it results $d b+d w \geq d b w$. In both cases, the ratio $d b w /(d b+d w)$ can be used as a multiplicative correction factor for the arithmetic mean $d b$ so as to fix a non ambiguous and proper value for the binarization threshold $\mathrm{T}$. Regions of $W$ with representative colors having distance from black smaller than $\mathrm{T}$ are assigned to the foreground of $W_{(0,1)}$. All other regions are assigned to the background.

The image $W_{(0,1)}$, binarized version of $W$, is refined by changing the status of some foreground regions into the status of background regions. To this aim, let us compute the average color $c_{F}$ of the foreground by averaging the representative colors of the regions assigned to the foreground of $W_{(0,1)}$, and the average color $c_{B}$ of the background by averaging the representative colors of the regions assigned to the background. Any region $R_{i}$, such that the distance between $c_{i}$ and $c_{B}$ is not larger than the distance between $c_{i}$ and $c_{F}$ is assigned to the background. At the end of this process the final binarized image $W_{(0,1)}$ is obtained.

The binarization resulting for the running example is shown in Fig. 4 left.
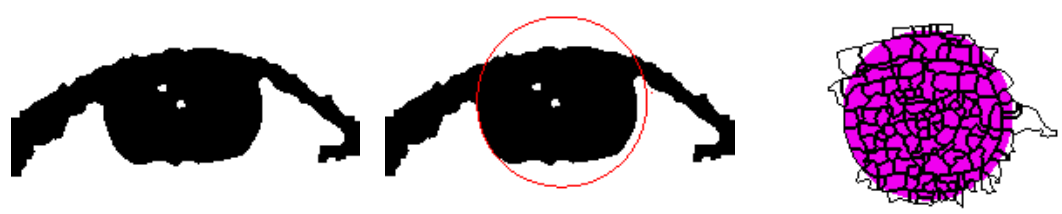

Fig. 4. Binarized version of the watershed transform, left, result of circle fitting on the binarized watershed transform, middle, and watershed regions at least partially overlapping the circle, right

Once the watershed transform has been binarized, a circle fitting process is taken into account to identify as precisely as possible the iris boundary. To this aim, different methods can be followed, e.g., those based on the Hough transform [9], which are unfortunately affected by high computational cost. The complexity increases at a rate of $\mathrm{O}\left(\mathrm{A}^{\mathrm{m}-2}\right)$ with each additional parameter, where $\mathrm{A}$ is the size of the image space and $\mathrm{m}$ is the number of parameters. We follow here the circle detection procedure presented by Taubin in [10], which in turn is able to detect circular shapes in an image precisely and reasonably fast. Indeed, when the shape is represented by a polynomial of degree $d$ it only analyzes $n \leq(2 d-1)$ moments. The overall computational complexity is $\mathrm{O}\left(3 h^{3}\right)$, where $h=\mathrm{O}\left(d^{n}\right)$. We do not describe in full detail the adopted circle fitting procedure (more details can be found in [12]) and limit ourselves to point out the main tasks faced for circle fitting. First, to save 
computation time, we focus the attention on contour information. In particular, we perform a curvature analysis with the aim of splitting the edges separating the iris from the sclera and eyelids wherever sharp changes in contour direction occur. Connected components including a small number of contour points are disregarded so as to reduce the number of contour components to which the Taubin algorithm is applied. Only circles completely included in the image are considered as of interest and the circle that has the highest score in a voting procedure is taken as delimiting the iris. See Fig. 4 middle.

\subsection{Iris Detection}

The watershed transform $W$ is used again in conjunction with the circle detected by means of circle fitting with the aim of better identifying all pixels of the input image really belonging to the iris. In fact, though the circle fitting process originates circles that delimit correctly the boundary between iris and sclera, it happens that the detected circular region includes parts of the image that belong to the eyelids or eyelashes rather than to the iris. Moreover, sometimes the shape of the iris is a bit more elliptical than circular and the detected circle has a radius slightly larger than the radius of the iris.

The watershed transform has all the info for solving the above problems. In fact, the watershed lines are found where high gradient values exist and hence in presence of significant changes of brightness.

Of course, the only regions of the watershed transform that should be taken into account for a better iris detection are those having an at least partial overlapping with the detected circle. These regions are shown in Fig. 4 right.

The representative color for the iris, $c_{i r i s}$, is computed as the arithmetic mean of the colors of the pixels of $I r$ that are within the circle and that in $W_{(0,1)}$ have been assigned to the foreground. In turn, the representative color for the background, $c_{b a c k}$, is computed as the arithmetic mean of the colors of the pixels of $I r$ that are outside the circle and that in $W_{(0,1)}$ have been assigned to the background.

In the iris segmented image, each region $R_{i}$ of $W$ will be associated a marker, which is either $f$ or $b$, depending on whether the region is assigned to the foreground or to the background.

Initially, regions completely overlapping and partially overlapping the circle are associated a temporary marker $f+$ and $f$ - respectively. These regions tentatively belong to the foreground, but need some further checking to decide. All other regions are associated the marker $b$, as they are directly classified as belonging to the background.

A region $R_{i}$ with marker $f+$ is classified as belonging to the foreground (i.e., the marker $f+$ is changed into $f$ ) if any of the following conditions is satisfied:

1) the distance between the representative color $c_{i}$ and $c_{\text {back }}$ is larger than the distance between $c_{i}$ and $c_{\text {iris }}$;

2) $R_{i}$ has not a neighboring region $R_{j}$ with marker $f_{-}$, it has been assigned to the foreground in $W_{(0,1)}$ and has at least a neighboring region with marker $f+$. 
The first of the two conditions above is quite obvious: a region whose representative color is less similar to the average color of the background than to the average color of the iris is classified as belonging to the iris. As for the second condition, even if the representative color of $R_{i}$ is closer to the average color of the background, which would justify classification of $R_{i}$ as a background region, the fact that it has been assigned to the foreground in the binarized version of the watershed transform coupled with the fact that $R_{i}$ is surrounded by regions that are not all only partially overlapping the circle suggests to classify it as a foreground region.

As a result of the above process a number of regions with marker $f+$ have been assigned to the iris. These include regions for which distance between $c_{i}$ and $c_{\text {back }}$ is smaller than the distance between $c_{i}$ and $c_{\text {iris }}$. For any possibly remaining region $R_{i}$ with marker $f+$, the following process is accomplished. Let $d \min$ be the minimum distance in color between $R_{i}$ and each of its adjacent regions already classified as belonging to the foreground. If the distance between $c_{i}$ and $c_{\text {back }}$ is not smaller than $d m i n$, the marker of $R_{i}$ is set to $f$. Otherwise, the marker of $R_{i}$ is set to $b$.

The rationale for the above criterion is that notwithstanding the representative color of $R_{i}$ is closer to the average color of the background than to the average color of the iris, which would justify classification of $R_{i}$ as a background region, $R_{i}$ results to have a neighboring region, already accepted as a foreground region, to which the color of $R_{i}$ is closer than to the average color of the background.

The only regions for which a decision has to be taken are those partially overlapping the circle, characterized by marker $f$-. These regions are split into two sub-regions that overlap completely the circle and do not overlap at all the circle, respectively. The sub-regions receive marker $f+$ and $b$ respectively. After subdivision, the quantized image is updated. Each region $R_{i}$ with marker $f+$ is classified as belonging to the foreground if there exists at least a neighboring region already classified as belonging to the foreground and either the distance between the representative color $c_{i}$ and $c_{i r i s}$ is smaller than the distance between $c_{i}$ and $c_{b a c k}$, or the distance between $c_{i}$ and $c_{b a c k}$ is smaller than the minimum distance between $c_{i}$ and the color of its adjacent regions with marker $f$. Otherwise, region $R_{i}$ is classified as belonging to the background.
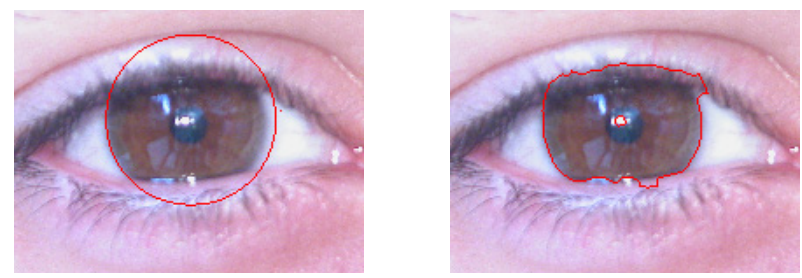

Fig. 5. The circle found by circle fitting, left, and the iris boundary detected by our procedure, right

The result of the process is shown in Fig. 5. In particular, the circle obtained from circle fitting is shown superimposed on Ir in Fig. 5 left, while the contour of the region detected as iris by our procedure is shown superimposed on Ir in Fig. 5 right. 
It can be easily observed that our procedure identifies much better the pixels actually belonging to the iris. It can be noted that the iris boundary detected by our process is significantly closer to the real iris boundary than the circle found by means of circle fitting. For example, the radius of the circle (Fig. 5 left) is slightly larger than the actual radius of the iris and we note that the boundary detected by our process (Fig. 5 right) better fits the boundary between iris and the part of the sclera to the right. Moreover, it is easy to note that a number of pixels actually belonging to the eyelids are placed in the top and bottom parts of the circle (Fig. 5 left), while most of them are not classified as belonging to the iris by our procedure (Fig. 5 right). A few more results for different color and opening of eyes and for different light conditions, are shown in Fig.6. The circle found by circle fitting and the iris boundary detected by our procedure are shown in green and red color, respectively.
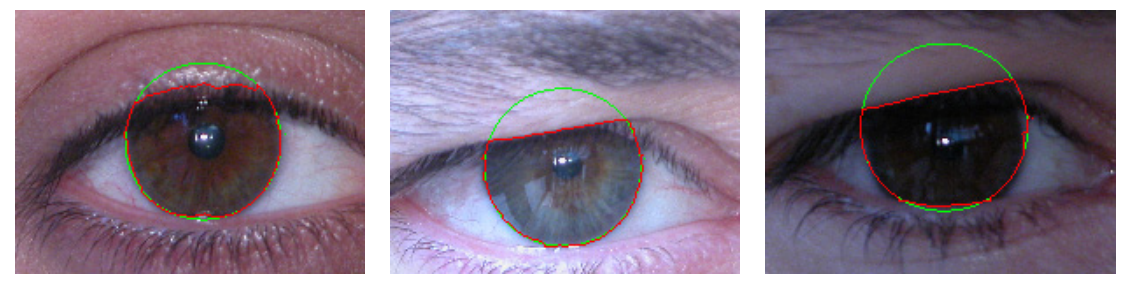

Fig. 6. The (green) circle found by circle fitting, and the (red) iris boundary detected by our procedure superimposed onto the image

\section{$4 \quad$ Experimental Setup and Results}

We have tested our iris detection method on the whole UBIRIS database obtaining generally rather good results. To make an evaluation of the precision of our iris detection method, we have compared our results with those provided by two further iris segmentation approaches. The former is ISIS [12]. The latter is a system built from Masek's implementation [11] of various iris-related algorithms, where segmentation is performed according to Wildes' method. Moreover, we also compared our results with a ground truth where each iris segmentation has been obtained manually.

The parameter having a major impact on the performance is the similarity between the contour made by automatic detection and the manually one. The X-axis and $\mathrm{Y}$-axis distances are the measure of dissimilarity and are computed as the average (mean) of the differences in absolute value between the respective coordinates of the contour points. Finally, as additional dissimilarity measure, the average value of the Euclidean distance between the respective couple of the corresponding contour points is computed. The summary of the performance of the indicators for the three compared techniques is shown in Table1. Each cell contains two values for $\mathrm{X}$-axis, Y-axis and Euclidean Distances: the top one is the mean value, whereas the bottom value is the standard deviation. 
Experimental results demonstrated that if the distances are greater than a 20 pixels threshold, the iris can't be detected because the eye is closed or the detection is wrong. In Table 1, the columns "no out." ("no outliers") report mean and variance computed by eliminating completely wrong locations (error greater than 20 pixels) or also UBIRIS closed eyes. Under the method's label we report the number of eliminated outliers.

Table 1. Location results for UBIRIS database

\begin{tabular}{|c|c|c|c|c|c|c|c|}
\hline \multirow{2}{*}{\multicolumn{2}{|c|}{ UBIRIS v1s2 }} & \multicolumn{2}{|c|}{$\mathrm{X}$ axis } & \multicolumn{2}{|c|}{ Y axis } & \multicolumn{2}{|c|}{ Euclidean } \\
\hline & & all & no out. & all & no out. & all & no out. \\
\hline $\begin{array}{c}\text { ISIS } \\
\text { out. }=8 \%\end{array}$ & $\begin{array}{c}\text { mean } \\
\text { std }\end{array}$ & $\begin{array}{l}2.01 \\
8.40\end{array}$ & $\begin{array}{l}0.042 \\
0.028\end{array}$ & $\begin{array}{l}14.38 \\
12.48 \\
\end{array}$ & $\begin{array}{l}5.97 \\
3.41 \\
\end{array}$ & $\begin{array}{l}14.33 \\
19.63 \\
\end{array}$ & $\begin{array}{l}6.37 \\
2.52 \\
\end{array}$ \\
\hline $\begin{array}{c}\text { WILDES } \\
\text { out. }=10 \%\end{array}$ & $\begin{array}{c}\text { mean } \\
\text { std }\end{array}$ & $\begin{array}{l}18.77 \\
10.21 \\
\end{array}$ & $\begin{array}{l}.143 \\
0.180 \\
\end{array}$ & $\begin{array}{l}27.36 \\
14.22 \\
\end{array}$ & $\begin{array}{l}8.345 \\
7.640 \\
\end{array}$ & $\begin{array}{c}32.82 \\
9.03 \\
\end{array}$ & $\begin{array}{l}9.250 \\
6.932 \\
\end{array}$ \\
\hline Proposed & mean & 1.03 & 0.037 & 4.89 & 3.857 & 5.44 & 3.881 \\
\hline $\begin{array}{c}\text { Method } \\
\text { out. }=4 \%\end{array}$ & std & 5.10 & 0.069 & 6.10 & 2.174 & 8.73 & 2.159 \\
\hline
\end{tabular}

The Table 1 shows that the best performance, i.e. minimum contour dissimilarity, was observed by $\mathrm{X}$-axis distance, since the methods using a circle to approximate the iris, as for example Wildes and ISIS, perform a greater error along the Y-axis and then on cumulative distance, due to the presence of the eyelids and eyelashes. On the contrary as the proposed method separates the iris from the eyelid, it approximates more closely the contour of limbo, if it is completely visible (Figure 7). Finally the number of outliers is lower than with other methods, as the proposed method performs a greater robustness: combining colors and contours it is able to locate the limbo even under conditions of significant image distortions.
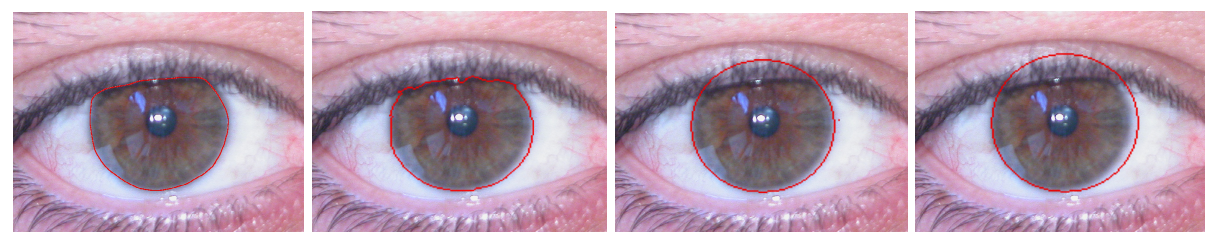

Fig. 7. The segmentation result provided by the four different approaches. From left to right: manual, the proposed approach, ISIS and WILDES.

\section{Conclusions}

A new iris segmentation method has been suggested for color images, based on the use of the watershed transform. The watershed transform is used in two phases of the process. First, it is used to obtain a binary version of the input image. Though, in this binary image the iris is not perfectly segmented, large portions of the iris boundary 
separating the iris from the sclera are clearly detectable. In this way, the circle fitting procedure aimed at localizing the iris is facilitated. Then, the watershed transform is newly analyzed in conjunction with the portion of the input image whose pixels are enclosed by the detected circle. In this way, it is possible to identify more precisely the pixels actually belonging to the iris. In fact, though the circle fitting process originates circles that delimit correctly the boundary between iris and sclera, it happens that the detected circular region includes parts of the image that belong to the eyelids or eyelashes rather than to the iris.

The method has been tested on a large database of images, producing generally rather satisfactory results both in terms of iris localization and segmentation. The method has also been compared to other methods in the literature and the obtained results show that our method is characterized by better performance.

\section{References}

1. Special Issue on the Segmentation of visible wavelength iris images captured at-a-distance and on-the-move. Image and Vision Computing, 28 (2010)

2. Special Issue on the Recognition of visible wavelength iris images captured at-a-distance and on-the-move. Pattern Recognition Letters, 33 (2012)

3. Wildes, R.: Iris recognition: an emerging biometric technology. Proceedings of the IEEE 85(9), 1348-1363 (1997)

4. Daugman, J.G.: How iris recognition works. IEEE Trans. Circuits and Systems for Video Technology 14(1), 21-30 (2004)

5. Puhan, N.B., Sudha, N.: A novel iris database indexing method using the iris color. In: Proc. of the IEEE Conference on Industrial Electronics and Applications, pp. 1886-1891 (2008)

6. Proença, H., Alexandre, L.A.: UBIRIS: A noisy iris image database. In: Roli, F., Vitulano, S. (eds.) ICIAP 2005. LNCS, vol. 3617, pp. 970-977. Springer, Heidelberg (2005)

7. Beucher, S., Lantuejoul, C.: Use of watersheds in contour detection, in. In: Proc. Int. Workshop on Image Processing, Real-Time Edge and Motion Detection/Estimation, France (1979)

8. Roerdink, J.B.T.M., Meijster, A.: The watershed transform: definitions, algorithms and parallelization strategies. Fundamenta Informaticae 41, 187-228 (2001)

9. Ballard, D.H.: Generalizing the Hough transform to detect arbitrary shapes. Pattern Recognition 13(2), 111-122 (1981)

10. Taubin, G.: Estimation of planar curves, surfaces and nonplanar space curves defined by implicit equations, with applications to edge and range image segmentation. IEEE Trans. on PAMI 13, 1115-1138 (1991)

11. Masek, L., Kovesi, P.: MATLAB Source Code for a Biometric Identification System Based on Iris Patterns. The School of Computer Science and Software Engineering (2003)

12. De Marsico, M., Nappi, M., Riccio, D.: IS_IS: Iris Segmentation for Identification Systems. In: 20th International Conference on Pattern Recognition, pp. 2857-2860 (2010) 